\title{
11
}

\section{Feedback \& Pricing in ATM Networks}

\author{
L. Murphy \\ Department of Computer Science and Engineering, \\ Auburn University, AL 36849, USA. \\ tel: +1 334 844-6326; fax: +1 334 844-6329 \\ email: lmurphy@eng.auburn.edu
}

\author{
J. Murphy \\ School of Electronic Engineering, \\ Dublin City University, Glasnevin, Dublin 9, Ireland. \\ tel: +353 1 704-5444; fax: +35317045508 \\ email: murphyj@eeng.dcu.ie
}

\begin{abstract}
Admission control and congestion control can provide traffic guarantees in ATM networks. However some users may not be able to describe their traffic accurately enough for the network to provide such guarantees. By sending a dynamic feedback signal about the current utilisation of network resources, the network could provide loss guarantees to users who respond appropriately, even without prior traffic descriptors. One possible feedback signal is a price per unit of network resource, based on the network load level : when the load is high, the price is high, and when the load is low, the price is low or zero. We outline a distributed iterative pricing algorithm, and show through simulations that it can simultaneously increase both network and economic efficiency. We also explore some arguments often raised against usage--sensitive pricing, and provide some counterarguments.
\end{abstract}

\section{Keywords}

ATM Networks, Pricing, Dynamic Feedback, Congestion Control

\section{INTRODUCTION}

Asynchronous Transfer Mode ( ATM ) has been adopted as the transfer mode for the Broadband Integrated Services Digital Network ( BISDN ), e.g. de Prycker (1993), a service-independent network capable of supporting all the communication services that users now require or may require in the future. ATM is also emerging as a local area net- 
working technology, since it provides flexible bandwidth-on-demand and internetworking capabilities for conventional data communications. ATM networks are therefore expected to accommodate a wide range of users, including some whose applications require guarantees on cell loss and/or delay. These guarantees could be deterministic worst-case or less stringent statistical guarantees. Some users may be satisfied with best-effort service, for which the network offers no guarantees on loss or delay.

Admission control and congestion control can provide performance guarantees and are therefore two of the most important ATM network functions. In order to obtain these guarantees from the network, users have to describe their traffic inputs by specifying values for network-defined traffic descriptors such as peak cell rate ( PCR ) or sustainable cell rate ( SCR ). However some users may not be able to describe their traffic accurately : because their applications cannot be sufficiently well-characterised by the given traffic descriptors, or because their actual traffic inputs depend on factors outside user control ( such as the number of active applications competing for access to a server ). A common assumption in many proposed admission control schemes is that traffic which is not well-described cannot get specific guarantees beyond the level of service being provided to best-effort traffic.

The ATM Forum has recognised the problem of providing guarantees to users whose traffic cannot be well-described, and in response has developed a specification for Available Bit Rate ( ABR ) service, e.g. Ramakrishnan (1995). Users who choose ABR service receive feedback from the network about the current level of network resource utilisation, and can get cell loss guarantees ${ }^{1}$ if they respond appropriately - by reducing their input rates in times of congestion, for example.

ABR service is therefore suitable for users whose applications are flexible with respect to delay but not necessarily to loss. This flexible behaviour represents a tool that network operators can use to increase network utilisation while continuing to serve guaranteed traffic such as CBR and VBR applications. In addition, this type of network feedback could modify an adaptive user's traffic at the source rather than after it has been injected into the network. This would help to localise the effects of feedback to the edges of the network and allow simpler internal network operation.

Most suggestions for supporting ABR service assume that well-described traffic which requires performance guarantees gets priority in the use of network resources such as bandwidth or buffer space, and that the remaining resources are fairly shared among the ABR users. Two issues which are not explicitly addressed are

- why more "demanding" traffic should get priority over ABR traffic;

- what constitutes "fair" sharing. Should the available bandwidth be shared equally among all ABR users, for instance? Or should it be shared according to the various application requirements?

\footnotetext{
${ }^{1}$ No specific delay guarantees can be provided, so ABR users must be prepared to absorb delays at the traffic source before being allowed to input traffic into the network.
} 
It is important to note that, just because such issues are not addressed explicitly, does not mean that these suggestions are neutral on what are often regarded as policy issues. On the contrary : sharing the available bandwidth equally among all ABR users values all such traffic equally, although the users themselves may put widely differing values on their service; giving CBR and VBR users priority over ABR users ignores the possibility that ABR users may value network access more than users with well-described traffic sources. We are not saying that these assumptions are wrong or undesirable, but instead we advocate allowing the users themselves to resolve these issues.

Admission control and congestion control in ATM are difficult problems which so far have not been satisfactorily solved. Two key questions are

- how should congestion be defined and measured ? This is a difficult question because individual user requirements vary considerably, so that one user may think the network is congested while another does not; and because in internetworks the responsibility for detecting congestion may be distributed among several network operators, each of which applies a different test at their bottleneck points.

- how should limited resources be allocated under congestion ? Some proposals call for users to indicate the relative priority of their traffic - leading to the problem of providing incentives so that all users will not choose the highest priority.

Our aim in this article is to propose a dynamic feedback control scheme which explicitly addresses these issues.

\section{DIFFERENT TYPES OF EFFICIENCY}

A network is as good, or as bad, as its users perceive it to be. This leads to the conclusion that network performance should be measured in terms of overall user satisfaction with the service they receive. Network engineering measures ( such as average packet delay or loss rate ) are inadequate reflections of user satisfaction when user requirements vary widely.

Due to the difficulty in accounting for individual user's requirements, however, aggregate network-oriented performance measures are usually used in design and operations problems. Usage is divided into classes according to application requirements and traffic characteristics; for example, real-time video, real-time audio, or off-line file transfer. Each class is regarded as having a single representative user for analytical and control purposes, and class objectives are used to drive the network control process. Therefore the loop is not closed all the way to the users when making operational decisions.

We propose to bring the users back into the loop and thereby ensure that performance measures are user-oriented, as shown in Figure 1. A user-oriented network control scheme would take user valuations into account : the network could serve higher-value users even under congestion by temporarily denying access to lower-value users. Such time- 
smoothing would not upset users who can tolerate longer delays, while it would improve the network's value to users who get greater benefits from immediate access.

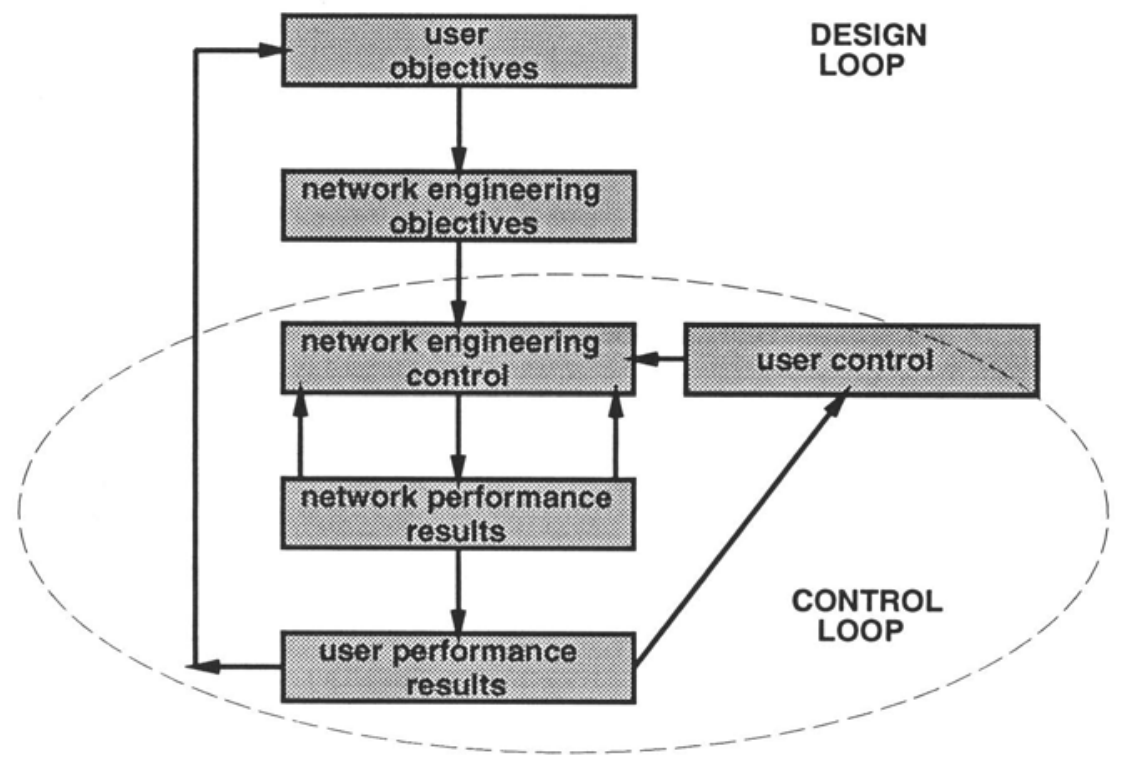

Figure 1 Network design and control loops.

Each user privately decides how much they value network access; our scheme involves giving them incentives to do this. Users would gain by obtaining service more closely matched to their needs; network operators would gain through improved network utilisation and increased user satisfaction with the service they receive. We hope to achieve the same (or better) network performance as with conventional congestion control and resource allocation schemes, while at the same time increase the total value of the network from the users' point of view. Network engineering measures will continue to be important, but we believe that user preferences should be the primary consideration driving resource allocation and congestion control schemes.

We need to distinguish two very different notions of efficiency :

- Network efficiency refers to the utilisation of network resources such as bandwidth and buffer space.

- Economic efficiency refers to the relative valuations the users attach to their network service.

If a network can maintain an acceptable level of service while minimising the resources necessary to provide this service, we say that its operation is network efficient. If no user 
currently receiving a particular Quality of Service ( QOS ) values it less than another user who is being denied that QOS, we say that operation is economically efficient.

An obvious question is, why will either type of efficiency continue to be important ? Some observers have suggested that the widespread deployment of fibre optic lines, and continuing exponential decreases in processor and memory costs, will result in these network resources becoming essentially "free" so that efficiency in their use will not be important in the future, and all users can always be accommodated. We do not believe these arguments apply in the short or medium terms, if indeed they will ever apply. User demands are increasing exponentially, so that it is not clear when - if ever - network resources will be "free". Experience suggests that application developers will have no difficulty in designing new services that use up all available resources, perhaps after an initial adjustment period. And market economics dictates that commercial network operators should be aware of the differing valuations that users attach to the same level of network performance. The same considerations apply to privately owned or operated networks : the ultimate goal will continue to be to maximise some measure of the value of using the network.

\subsection{IMPROVING EFFICIENCY WITH FEEDBACK}

Users with flexible traffic inputs can help to increase network efficiency if they are given appropriate feedback signals. When the network load is high, the feedback should discourage these users from inputting traffic; when the load is low, the feedback should encourage them to send any traffic they have ready to transmit. Instead of regarding their load as fixed, the network uses the flexibility of these users as part of a congestion control and avoidance strategy. One possible feedback signal is a price based on the level of network load : when the load is high, the price is high, and when the load is low, the price is low or zero.

Similarly, by associating a cost measure with network loading, all users can be signalled with the prices necessary to recover the cost of the current network load. Price-sensitive users - those willing and able to respond to dynamic prices - increase economic efficiency by choosing whether or not to input traffic according to their individual willingness to pay the current price. Users who value network service more will choose to transmit, while those who value it less will wait for a lower price.

Price signals thus have the potential to increase both network and economic efficiency, though whether a particular pricing scheme increases either notion of efficiency depends on the implementation. One important point needs to be clarified :

- contradictory though it sounds, a scheme based on pricing principles does not necessarily involve money. For example, in a private network where one organisation controls all the users, or in a company's virtual private network, the "prices" are simply control signals. In this case, the users' applications could be programmed to obtain a desirable traffic mix, to enforce priorities, or to achieve 
some other objective.

We envisage that the charge to a user in an ATM network might have many components, such as a connection fee, a charge per unit time or per unit of bandwidth, premium charges for certain services, and so on. We suggest that there should also be a usage-sensitive component during congestion, to increase both network and economic efficiency. We propose charging only when network congestion indicates that some users may be experiencing QOS degradation, with the size of the charges related to the degree of congestion. If the network is lightly loaded and all users are getting acceptable QOS, the usage-sensitive prices would be zero.

We recognise that many people are concerned about the use of pricing in network operations. Concerns range from questions about the feasibility and overhead of usage-sensitive pricing, to policy issues such as profit opportunities and fairness. We believe that a clear understanding of the nature of what is being proposed is necessary on all sides. Therefore we first outline our proposed dynamic pricing scheme and some preliminary simulation results, and then address some of the objections often raised in discussions of dynamic network pricing.

\subsection{DISTRIBUTED ITERATIVE PRICING ALGORITHM}

It is important to note that our proposed pricing algorithm would only be applied to adaptive users, who are able and willing to respond to dynamic prices during a connection by changing their offered traffic. All other users would be charged according to another pricing scheme. How to co-ordinate the various pricing schemes to achieve some overall objective ( such as fairness ) is a complex issue and we do not address it in this article.

The network and its users are considered to form an economic system. The system has various resources such as link bandwidths and buffer spaces that can be used to meet user demands for service. Network constraints such as buffer sizes or link capacities are translated into cost functions on the demands for resources. The basic property of these cost functions is that marginal cost should go to infinity as usage of the resource approaches capacity.

Each adaptive user is viewed as placing a benefit, or willingness-to-pay, on the resources they are allocated. Given a price per unit of bandwidth or buffer space, a user's benefit function completely determines that user's traffic input. A benefit function could follow the usual economic assumption of diminishing incremental benefit as more of the resource is consumed, see Figure 2(a). Or the user could apply a threshold rule, or series of threshold rules, for deciding how much of the resource to request based on the current price, see Figure 2(b),(c). 


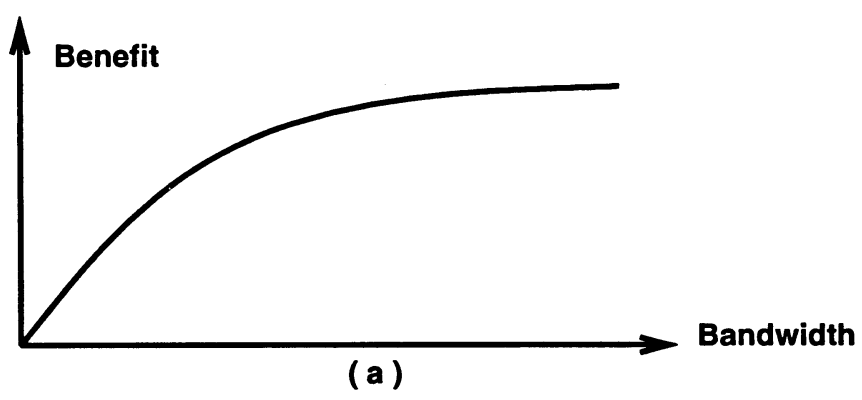

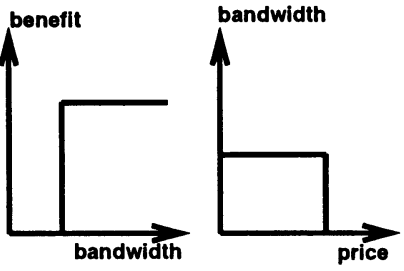

(b)

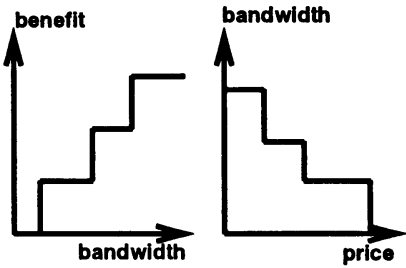

(c)

Figure 2 Possible user benefit functions.

The network operator sets the prices so that the marginal benefit the users place on their resource consumption is equal to the marginal cost of handling the resulting traffic in the network ${ }^{2}$. The network operator dynamically adjusts the prices based on current network conditions. It turns out that it is not necessary for the network operator to know the user benefit functions; therefore our pricing scheme is suitable for public as well as private networks.

Time is divided into feedback intervals, within each of which the prices and user benefits are fixed. This model allows users to potentially change their benefit functions every feedback interval, to reflect their satisfaction with the level of service received or their time constraints on having their cells accepted into the network, so the examples in Figure 2 are for a particular interval. Similarly the network re-calculates the prices every feedback interval to reflect current resource usage ${ }^{3}$.

A distributed iterative pricing algorithm for adaptive users has been developed, e.g. Murphy (1994), Murphy and Posner (1994), see Figure 3. The computation required per iteration at each user and ATM access switch is simple, which suggests that inexpensive processing elements may be sufficient in executing the algorithm.

\footnotetext{
${ }^{2}$ These prices only address the variable costs corresponding to network constraints.

${ }^{3}$ The network and the users may use prediction in their decisions without invalidating this model.
} 


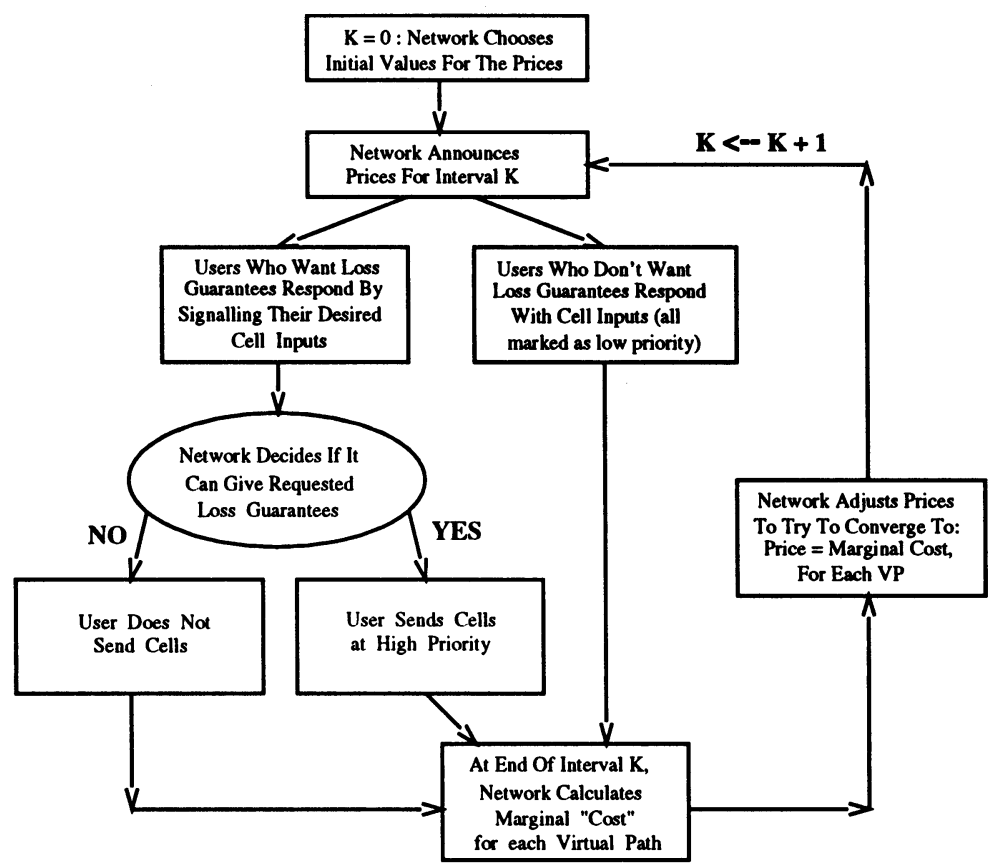

Figure 3 Distributed iterative pricing algorithm for adaptive users.

There are several different types of adaptive users, e.g. Murphy (1995), depending on whether the user is flexible with respect to loss, delay, or both. So far we have modelled two types of adaptive user :

- Inelastic. This user requires a delay bound on their traffic, but can tolerate sending only a fraction of the cells that are ready to transmit in the current interval. We assume that cells not sent in the interval are useless to the user and are discarded. For example, this might be the second level of a two-level video codec. The first level contains the minimum necessary information, and would be transmitted as a non-adaptive application. The second level consists of enhancement information. It is not essential that all of the information be delivered; however, a delay guarantee is required - if the enhancement information does not arrive before the playback point, it is considered useless.

- Elastic. This type of user waits until feedback from the network indicates that they can input traffic, then transmits and requires that their cells are not lost in the network. Each elastic user decides individually what their transmission criteria are, e.g. the maximum price per cell they are willing to pay. An example of an elastic user type would be a non-real-time data transfer with no ARQ capability, where already-transmitted cells are not buffered at the sender. 
The mathematical models used for inelastic and elastic users are described in Murphy (1996), along with the equations governing their responses to the dynamic prices from the network.

\section{SIMULATION MODELS \& RESULTS}

The simulated network is a high-speed ATM 155 Mbps link shared by inelastic and elastic users. Video sources are modelled as inelastic users; data sources are modelled as elastic users. The link model is shown in Figure 4. The network and source models were simulated using SES/workbench, e.g. SES (1992), a discrete-event simulator that allows hardware and software simulation. The models were mainly created by use of its graphical user interface. SES/ workbench compiles the graphical code to $\mathrm{C}$ and creates an executable. The simulation execution platform was a cluster of Sparc-10 workstations.

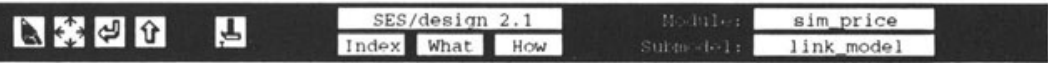
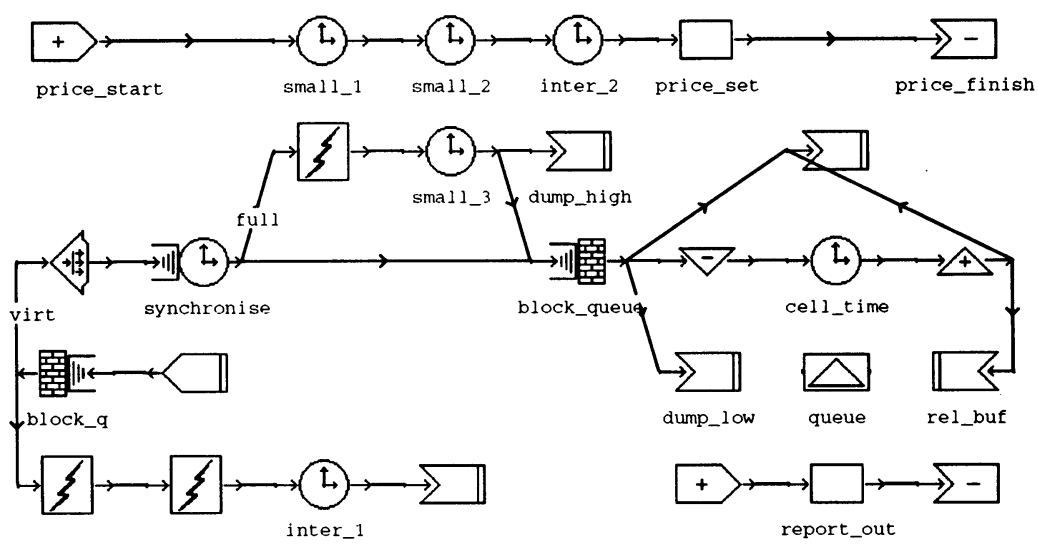

Figure 4 Simulation model for economic efficiency.

The simulation model is made up of submodules, each of which performs a well defined function. The sources generate cells which are input to a network interface submodule. The network interface takes the source bit stream and forms ATM cells. The cell stream from an interface is then input to the ATM switch buffer submodule. This submodule smooths the arrival of cells to the ATM network and so takes care of cell scale congestion. The switch buffer is the limited network resource in our model.

The video source model that we use here is a standard one for video conferencing, e.g. COST (1993). The codec has a compressed bit rate of $2.3 \mathrm{Mbps}$, which adheres to the 
H.261 standard for video, e.g. Murphy and Teahan (1994). There are 20 video sources, each with a mean of $2.3 \mathrm{Mbps}$ and a peak of $5 \mathrm{Mbps}$. These are input at a rate of 30 frames per second, all synchronised together, so that the inelastic users are (more or less ) stationary on the millisecond scale.

The elastic users can be thought of as one user with a lot of files to transfer independently, many users each with one file, or some combination of these types. The negotiations for file transfer or connection set up will only occur when a new video frame is to be sent, i.e. every $1 / 30$ second, because this simplifies the simulation and makes it possible to speed up the run time. Therefore the network renegotiates the PCR every $1 / 30$ second with the elastic users. An empirical distribution for file size ranges was obtained from actual files stored on one of our computers. In the simulations a range was chosen according to this empirical distribution, and then a file size was chosen from a uniform distribution within this range. The peak-to-mean ratio of this source can be high with values up around 1000. The number of data sources in use is taken from a uniform distribution between 1 and 39 sources. Each file to be sent is taken from a uniform distribution between 20 and 660 cells. The average bit rate of a single data source is therefore about $4.3 \mathrm{Mbps}$.

In our proposed scheme a price is generated by the network based on the present state of the network buffer, and the sources adapt their demands based on this price. What we propose and simulate adheres to the UNI 3.0 specification from the ATM Forum, e.g. ATM Forum (1993). A leaky bucket is also implemented on top of our scheme so that if there is cell loss we can discard the marked ones first.

The model takes in cells over a feedback interval and gives a price to all the sources sharing the link. The price reflects the congestion (if any ) in the buffer and hence on the virtual path. The feedback interval is short compared to the video frame time : a value of about 0.05 of a frame time was chosen. To achieve feasible run times we neglect cell scale effects. This neglecting of cell scale effects is critical to the speed up of the simulations. The total utilisation of the link is high, at a value of around 0.85 .

Our results are shown in Table 1 and show the difference between using pricing and no pricing. What can be seen is that both network efficiency and economic efficiency increase at the same time by using pricing. Cell loss drops from $19 \%$ to under $2 \%$, while the net benefits perceived by the users increase by nearly $15 \%$.

Table 1 Performance and Economic Gains from User Feedback.

\begin{tabular}{|l|l||c|c||c|c|}
\hline \multicolumn{2}{|c||}{ Source Type } & \% Loss & User Value & \% Dec. Loss & \% Inc. Value \\
\hline \hline \multirow{3}{*}{ Unpriced } & Inelastic & 0 & 240 & & \\
\cline { 2 - 4 } & Elastic & 30.4 & 146 & & \\
\cline { 2 - 4 } & Combined & $\mathbf{1 9 . 1}$ & $\mathbf{3 8 6}$ & & \\
\hline \multirow{3}{*}{ Priced } & Inelastic & 4.4 & 239 & & \\
\cline { 2 - 4 } & Elastic & 0.1 & 204 & & \\
\cline { 2 - 4 } & Combined & $\mathbf{1 . 7}$ & $\mathbf{4 4 3}$ & $\mathbf{9 1 . 0}$ & $\mathbf{1 4 . 8}$ \\
\hline
\end{tabular}




\section{CONCERNS ABOUT USAGE-SENSITIVE PRICING IN NETWORKS}

We explore some common arguments against usage-sensitive pricing in network operations in this Section, and provide some counter-arguments. Some previous work along these lines is contained in MacKie-Mason (1994).

- once a network is installed, any load-dependent costs of transferring data are minimal - the fixed costs of network management and maintenance dominate. These fixed costs can be efficiently recovered through connection fees and capacity prices. Why implement an elaborate pricing mechanism to recover the relatively small variable costs?

- Counterpoint : this ignores the congestion cost which one user's traffic imposes on other users sharing the resources. Bandwidth or buffer space occupied by one user's traffic is not available to other users. When this reduces other users' quality of service ( through increased delays, loss rates, blocking probabilities, and so on ), they suffer congestion costs which may translate into significant actual costs of service degradation. One mechanism to capture these costs is a price which is sensitive to some indicator of congestion, such as load.

- even if we want to consider congestion costs, how can the network determine what actual costs the current load is imposing on users who probably have widely varying service requirements? Getting users to reveal these costs is likely to be extremely complicated, if not impossible.

- Counterpoint : it is true that providing users with the right incentives to reveal their actual costs of service degradation is complicated. However, with any prices that increase with the degree of congestion in the network, users will be induced to prioritize their traffic. Only users who value their traffic at least as much as the current price will transmit. If congestion remains unacceptably high, then the price was too low; conversely if capacity is unacceptably underutilized, the price was too high. Thus, through a process of experimentation and dynamic adjustment, the network can shape the price schedule so that users approximately reveal their valuations for uncongested service through their responses to the prices.

- why won't some non-pricing scheme be enough ? Administrative controls can be used to impose some appropriate notion of fairness, for example; or users can choose a traffic priority level which matches their requirements.

- Counterpoint : who decides what is fair ? The network operator can; but according to a user-oriented objective, fairness should be determined collectively by the users. We might all agree that telesurgery is more important than email, but what about interactive video games versus email ? Also, every time a new application is developed it has to be slotted into the priority order, an increasingly complex process. Suppose the network simply supports priority levels and allows each user to choose their own level. Why wouldn't 
they all choose the highest priority ? To guard against such abuses, there would have to be some penalty for "inappropriate" declarations, implying the need to define "appropriate" priority levels or to assign increasing charges to higher priorities, e.g. Bohn (1993). A user's choice of priority level would then be based on economic considerations : balancing the benefits of higher priority against the costs and/or the penalties for inflating their application's perceived priority level. Pricing represents the limiting case of a continuous spectrum of priorities.

- most users will want to know their charges in advance, and will not want to deal with prices that change during the lifetime of a typical connection.

- Counterpoint : we are not advocating that all users must face usage-sensitive prices. Any user can choose not to face dynamic prices, even if their application is adaptive. They would then be charged according to some other pricing scheme, which should be co-ordinated with the usage-sensitive pricing mechanism. Or a user faced with dynamic prices can choose to ignore those prices by transmitting at their application's natural information rate, and paying the resulting charges. Finally, in our scheme - and in any realistic pricing schemeit would be possible for a user to set the maximum charge they are willing to pay, which is what is usually required for budgetary purposes.

- bits/bytes/cells are not the correct units to charge for - it's information that users care about. Any scheme which proposes to look inside every data unit to determine how it relates to other data units is likely to be too complex to be justified. Also, lower-layer mechanisms ( such as Ethernet collisions ) or cell losses requiring higherlayer retransmissions make it difficult to predict how much "raw" data has to be transmitted to transfer a given amount of information. Should users be charged for retransmissions that they have no control over, or cells which are dropped by the network?

- Counterpoint : our scheme involves pricing for transport, not for content. The "importance" of a particular cell, and its relation to other cells, is a higherlayer issue determined by the application ( or ultimately by the users ). We are not proposing that the network be aware of these issues; on the contrary, the network view in our scheme is that it's up to the users to decide how cells are used to transfer information. It is in general impossible to predict exactly how many cells are required to transmit a block of information, but again this is a higher-layer issue. The basic question is whether the users or the network should bear the uncertainty. If the network is expected to offer a "file transfer" service, the file transfer charge per megabyte could be computed by averaging over many such transfers. If the user is expected to pay for all transmitted cells, their application could for example define a maximum number of cells they are willing to transmit per megabyte of information, or a maximum amount they are willing to pay per megabyte of information actually transferred. 
- dynamic pricing schemes are unworkable in practice due to the overheads involved in accounting and billing for usage on such a detailed level. In addition, a significant portion of the revenue raised is needed to defray the cost of doing dynamic pricing in the first place.

- Counterpoint : the costs of dynamic pricing may outweigh the benefits for a particular implementation but we do not believe this is necessarily true for all dynamic pricing schemes. In particular, online pricing mechanisms may reduce the actual cost to an acceptable level; there is no reason to think that current billing and accounting costs in other industries, such as telephone or electricity networks, will necessarily apply to dynamic pricing in ATM networks. This concern can only be answered for each scheme individually, and should obviously be part of the overall decision on what usage-sensitive pricing scheme to implement, if any.

- dynamic pricing is impractical because users cannot respond to prices which are updated many times per second. If the update interval is increased to the minimum period in which users can respond, congestion can arise and disperse in between price updates, so that prices no longer influence user behaviour.

- Counterpoint : our scheme assumes an intelligent network interface at pricesensitive user sites, so the processing necessary to respond to dynamic prices would be done automatically based on pre-programmed user preferences. Current ATM connection admission control schemes already assume enough user intelligence to be able to negotiate quality of service parameters, so our scheme adds a little more complexity rather than a new requirement. This software would play a similar role to current TCP implementations, which respond to network feedback by adjusting their traffic inputs, except that the feedback in our case is the current price.

- charging for cells transmitted fails to capture cases where the benefit of a transfer is with the receiver. If senders are charged for receiver-initiated transfers, we could see a drastic reduction in the number of open-access servers with a corresponding decrease in the value of using the network.

- Counterpoint : we do not believe that associating the charge for a transmission with the sender constrains the actual flow of money in any way. It is easy to imagine multiparty connection protocols which initially negotiate each party's responsibility for the total charge, or "reverse-charges" servers which only transmit data once the receiver has indicated willingness to pay the resulting transmission costs.

- With any form of usage-sensitive pricing, it's the small users who will suffer the most. Rich users could behave as they want since they have the resources, and could effectively limit the network access of smaller users.

- Counterpoint: with our scheme, if your application is flexible enough, your charges would be zero. Many opponents of usage-sensitive pricing seem to 
believe that it inherently involves charging for every cell/bit/etc. However our scheme explicitly recognises that if there is no congestion in the network, the usage-sensitive price should be zero. Users who are flexible enough to wait for such periods can then transmit for free $e^{4}$. It would be a relatively simple matter to set a maximum price per cell of zero and let your network interface determine when to transmit - assuming your application can wait for the price to drop. As for rich users being able to afford to ignore dynamic prices, this is true under any pricing scheme and is not particular to dynamic pricing. We do not mean to dismiss income distribution problems as unimportant, but merely to say that network pricing ( or non-pricing) is not the right venue for solving them.

- usage-sensitive pricing is just another way for network operators to make more money. Users will lose out as network operators maximise their profits.

- Counterpoint : it's true that there is the potential for profiteering whenever prices are charged, especially when the conditions under which prices are set are not immediately accessible to ordinary users. But in a competitive environment, network operators have market incentives to keep their margins of revenue over actual cost as low as possible. This incentive is missing in the case of a monopoly provider or a cartel of price-fixing providers. But whether abuse is possible in this case depends on policy and regulatory decisions rather than on the specific pricing scheme.

- economics is important in network planning but has nothing to do with the technical operation of a network, whether public or private.

- Counterpoint : economics has a lot to do with network operation! Packetswitching was developed for computer communications because around 1970 it became more economical to use switching and routing to statistically multiplex several connections into one transmission link, rather than dedicating one circuit to each connection as in circuit-switching. Economics plays a role in formulating and solving decision problems in all types of network; current price schemes differ from ours in the frequency with which prices are updated. Our scheme simply moves this updating into "real-time" for those users who are able and willing to respond on that timescale.

\section{CONCLUSIONS}

We have presented an economic framework for adaptive users in ATM networks. Instead of the typical requirement for traffic descriptors in order to get performance guarantees, these flexible users can get loss guarantees if they adjust their traffic input rates in response to dynamic feedback from the network. This is the basis for recent proposals for

\footnotetext{
${ }^{4}$ If the price is never zero, the network is always congested and capacity expansion is indicated.
} 
ABR service in ATM. Our framework takes these proposals one step further by explicitly defining how that feedback is generated by the network, and what form it takes. In our scheme the network associates a cost measure with the utilisation of network resources, announces a price which is based on the current cost, and price-sensitive users adjust their cell inputs based on this price and their own specification of how valuable network service is to them. While we address only the reactive control of adaptive users, our scheme could be part of a more comprehensive billing and accounting scheme to charge all users for network services.

What we propose is to give users incentives to consider the effects of their usage on other users. In a public network, where the users cannot be assumed to be cooperative, more traditional feedback schemes are not robust to user manipulation : it is relatively easy to program a host to ignore the feedback signals. Of course it would be just as easy to ignore price signals; but since users would be liable for charges they incurred, there is some incentive to respond.

We also address the problem of user service valuations, and allow for adaptive sources to have more demanding traffic than well-described sources. We have proposed a distributed iterative pricing algorithm and shown (by simulation ) that it is possible to gain both network efficiency and economic efficiency by using pricing. In other words, the network actually carries more traffic and carries more important traffic from the users' point of view.

\section{ACKNOWLEDGEMENTS}

We thank Jeff MacKie-Mason for many useful discussions about this work, and the contributors to the com-priv Internet mailing list for their objections to usage-sensitive pricing.

\section{REFERENCES}

ATM Forum (1993) 'ATM User-Network Interface Specification', Prentice Hall.

Bohn, R., Braun, H. W., Claffy, K. and Wolff, S. (1993) 'Mitigating the coming Internet crunch: Multiple service levels via precedence.' Tech. rep., UCSD, San Diego Supercomputer Center, and NSF.

COST (1993) 'Redundancy Reduction Techniques for Coding of Video Signals in MultiMedia Services', COST 211 ter, Compendium ATM, Coding Procedures, Simulation Subgroup, pp 1-83.

de Prycker, M. (1993) Asynchronous Transfer Mode : Solution for Broadband ISDN, 2nd Ed., Ellis Horwood. 
MacKie-Mason, J. and Varian, H. (1994) 'Some FAQs about Usage-Based Pricing', available from URL ftp://gopher.econ.lsa.umich.edu/pub/Papers/useFAQs.html

Murphy, J. and Murphy, L, (1994) 'Bandwidth Allocation By Pricing In ATM Networks', IFIP Transactions C : Communication Systems, No. C-24, pp. 333-351, available from

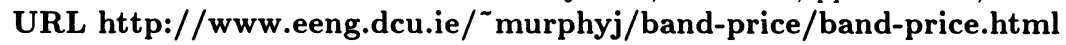

Murphy, J., Murphy, L. and Posner, E. C. (1994) 'Distributed Pricing For Embedded ATM Networks', Proc. International Teletraffic Congress ITC-14, Antibes, France, pp. 10531063, available from URL http://www.eeng.dcu.ie/ ${ }^{\sim}$ murphyj/dist-price/dist-price.html

Murphy, J. and Teahan, J. (1994) 'Video Source Models for ATM Networks', Eleventh UK IEE Teletraffic Symp., Cambridge, UK, available from URL http://www.eeng.dcu. ie/ ${ }^{\sim}$ murphyj/video/video.html

Murphy, L. and Murphy, J. (1995) 'Pricing for ATM Network Efficiency', Proc. 3rd International Conference on Telecommunication Systems Modelling and Analysis, Nashville, TN, pp. 349-356, available from URL http://www.eeng.dcu.ie/ ${ }^{\sim}$ murphyj/atmprice/atm-price.html

Murphy, J. (1996) Resource Allocation In ATM Networks, Ph.D. thesis, School of Electronic Engineering, Dublin City University, Ireland, available from URL file://ftp. eeng.dcu.ie/pub/tele-communications/murphy/thesis/thesis.ps

Ramakrishnan, K. K. and Newman, P. (1995) 'Integration of Rate and Credit Schemes for ATM Flow Control', IEEE Network, p. 49-56.

SES (1992) SES/workbench Reference Manual, Release 2.1.

\section{BIOGRAPHIES}

Liam Murphy is an Assistant Professor in the Department of Computer Science and Engineering at Auburn University. He received his $\mathrm{PhD}$ in Electrical Engineering from the University of California at Berkeley in 1992. His current research interests are in the areas of resource allocation and congestion control in integrated-services networks, and multimedia networking. He is a member of the IEEE.

John Murphy is a lecturer in the School of Electronic Engineering at Dublin City University, where he received his $\mathrm{PhD}$ in 1996. He has worked with AT\&T, Telecom Eireann and JPL on various teletraffic problems. His current research interests are in the areas of resource allocation in ATM networks and in modelling and simulation of high speed networks. He is a member of the IEEE. 